\title{
Prognostic Factor Analysis of Intraocular Pressure with Neovascular Glaucoma
}

\author{
Satoko Nakano, Takako Nakamuro, Katsuhiko Yokoyama, \\ Kunihiro Kiyosaki, and Toshiaki Kubota
}

Department of Ophthalmology, Oita University Faculty of Medicine, 1-1 Idaigaoka, Hasama-machi, Yufu-City, Oita 879-5593, Japan

Correspondence should be addressed to Satoko Nakano; sanakano@oita-u.ac.jp

Received 9 February 2016; Revised 18 May 2016; Accepted 30 May 2016

Academic Editor: Ciro Costagliola

Copyright (C) 2016 Satoko Nakano et al. This is an open access article distributed under the Creative Commons Attribution License, which permits unrestricted use, distribution, and reproduction in any medium, provided the original work is properly cited.

Purpose. To perform multivariate analysis for identifying independent predictors of elevated intraocular pressure (IOP) with neovascular glaucoma (NVG), including antivascular endothelial growth factor (VEGF) intravitreal injections. Methods. We retrospectively reviewed 142 NVG patients (181 eyes) with ischemic retinal diseases [proliferative diabetic retinopathy (PDR) in 134 eyes, retinal vein occlusion (RVO) in 29, and ocular ischemic syndrome in 18]. We analyzed age, gender, initial/final LogMAR VA, initial/final IOP, extent of iris and/or angle neovascularization, treatments, preexisting complications, concurrent medications, and follow-up duration. Results. The mean follow-up duration was $23.8 \pm 18.8$ months. At the final follow-up, 125 (72.3\%) eyes had IOP $\leq 21 \mathrm{mmHg}$. NVG patients with RVO had a higher degree of angle closure and higher IOP. NVG with PDR had better IOP and LogMAR VA. Angle closure had the greatest impact on final IOP. Greater than $90 \%$ of patients treated with trabeculectomy with mitomycin C (LEC) had persistent declines in IOP $(\leq 21 \mathrm{mmHg})$. Stand-alone and combination anti-VEGF therapies were not associated with improved long-term prognosis of IOP. Conclusions. Angle closure was found to have the greatest effect on NVG-IOP prognosis. When target IOP values are not obtained after adequate PRP with or without anti-VEGF, early LEC may improve the prognosis of IOP.

\section{Introduction}

Neovascular glaucoma (NVG) is a severe consequence of a number of ocular and systemic conditions, such as proliferative diabetic retinopathy (PDR), retinal vein occlusion (RVO), ocular ischemic syndrome (OIS), tumors, trauma, or uveitis [1]. It is indisputable that increased awareness of risk factors and the early detection of retinal ischemia can minimize poor prognosis of NVG; however, many NVG patients suffer loss of vision as a result of irreversible high intraocular pressure (IOP) despite the use of preexisting treatments, such as panretinal laser photocoagulation (PRP), pars plana vitrectomy (PPV), and trabeculectomy with mitomycin C (LEC) [2]. The main causative factor of NVG is retinal hypoxia that initiates the subsequent release of angiogenesis factors, and NVG is characterized by changes in the appearance of the iris, angle closure glaucoma, and formation of new vessels [1]. Gartner and Henkind showed that the main mechanism of intraocular pressure elevation was angle closure, with peripheral anterior synechia [3]. However, NVG occurs when new fibrovascular tissue proliferates onto the iris and chamber angle structures, including the trabecular meshwork, typically in response to ischemia of various etiologies $[2,4,5]$. Previous studies have demonstrated increased production of several proangiogenic factors, including vascular endothelial growth factor (VEGF) [6]. In addition to the ischemic retina, nonpigmented ciliary epithelium and iris contribute to VEGF synthesis in NVG patients [7]. The created fibrovascular neovascularizational membrane promoted by these proangiogenic factors inhibits the aqueous flow and leads to an increase in IOP [4]. Recent studies on intravitreal injection of anti-VEGF agents as stand-alone or as combination treatment with other NVG treatments have reported excellent results [8-12]; however, responses to treatment with a single injection are considered temporary $[13,14]$. Anti-VEGF treatment was introduced in our university hospital in September 2006. We compared the cause-specific prognosis of NVG and efficacy between 


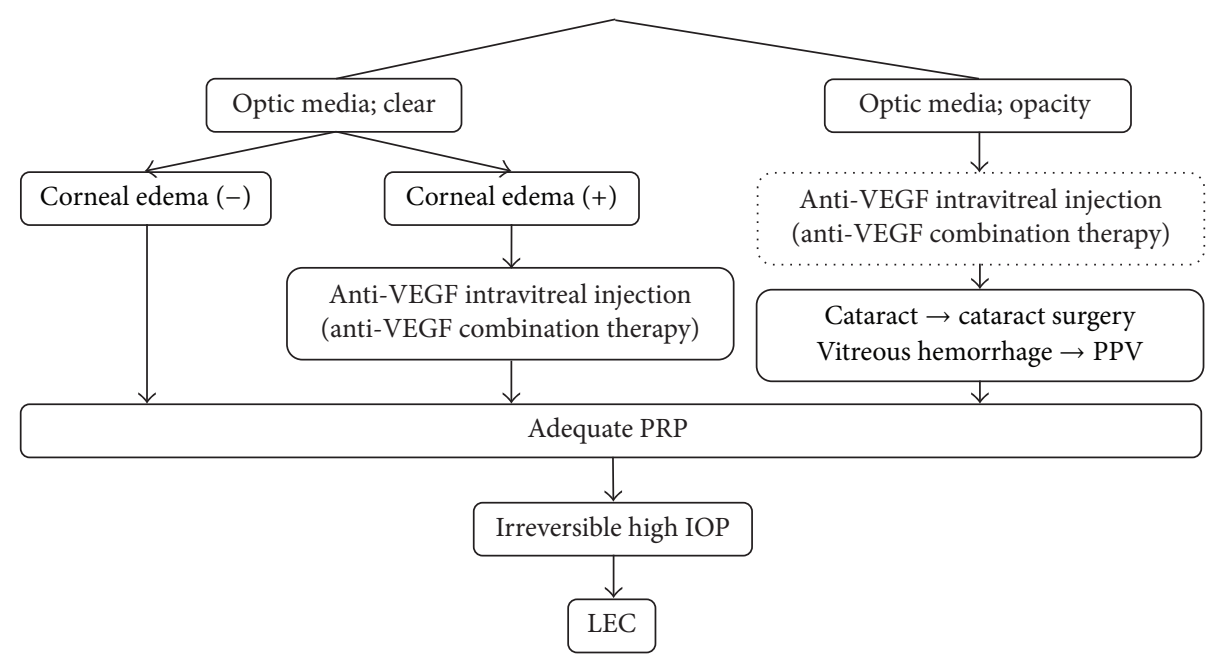

FIGURE 1: Treatment plan of NVG in Oita University Hospital. A variety of treatments were administered in the present study depending on NVG patient status, including panretinal laser photocoagulation (PRP), anti-VEGF intravitreal injection (stand-alone or in combination with other treatments), cataract surgery, pars plana vitrectomy (PPV), and trabeculectomy with $0.02 \%$ mitomycin C (LEC). PRP, panretinal laser photocoagulation; PPV, pars plana vitrectomy, LEC, trabeculectomy with mitomycin C; VEGF, vascular endothelial growth factor.

treatments. Further, we performed multivariate analysis to identify NVG prognostic factors for IOP.

\section{Subjects and Methods}

2.1. Subjects. We performed a retrospective study of $142 \mathrm{NVG}$ patients (181 eyes) with ocular ischemic diseases who had visited the Oita University Hospital between September 2006 and May 2014 (follow-up duration: mean \pm STD, $23.8 \pm$ 18.8 months; range: 4.0-81.1 months). All procedures were performed in accordance with the Declaration of Helsinki.

2.2. Diagnostic Criterion for NVG. All patients underwent full ocular examinations, including undilated gonioscopy and pupil examinations [2]. NVG was staged according to the degree of neovascularization, angle closure, and intraocular pressure (IOP). Anterior segment fluorescein angiography (FA) and indocyanine green angiography (IA) for iris and angle neovascularization were used to confirm the presence of newly formed vessels. NVG staging criteria in this study were as follows: rubeosis group, angle and/or iris neovascularization only without peripheral anterior synechia (PAS) and normal IOP; open-angle NVG group, open angle and high IOP ( $>21 \mathrm{mmHg}$ ) due to neovascularization; and angle closure NVG group, closed angle and high IOP $(>21 \mathrm{mmHg})$ with PAS.

2.3. Treatment Plan of NVG. NVG patients with clear optic media without corneal edema were treated with adequate PRP. Adequate PRP was defined as more than 3000 laser burns sufficient for disappearance of nonperfusion area (NPA). For patients with clear optic media and corneal edema, we performed adequate PRP after antiVEGF (bevacizumab [11, 14-18] or ranibizumab [8, 9, 19]) intravitreal injections $(0.5 \mathrm{mg} / 0.05 \mathrm{~mL})$. For patients with severe ischemic retinopathy, anti-VEGF was also injected in anticipation of NPA reduction after adequate PRP. Bevacizumab was used before the approval of ranibizumab. Patients administered bevacizumab were informed regarding the off-label use of these drugs in the majority of cases at the time of injection and the approval of their use by the Institutional Review Board of Oita University. Patients with optic media opacity were first treated with cataract surgery or PPV before adequate PRP. LEC was performed after adequate PRP in patients with high IOP. Anti-VEGF intravitreal injections were occasionally combined with the treatments listed above. Combination therapy was defined as single anti-VEGF intravitreal injection within 2 weeks of other treatments. A proportion of patients were treated with stand-alone anti-VEGF intravitreal injections according to patient preference when IOP elevation or neovascularization exacerbation was observed (Figure 1). Concurrent administration of medications, such as systemic acetazolamide and combination eye drops, with all other treatments was performed as required.

2.4. Observation Items. Observation items were age, gender, initial LogMAR VA (visual acuity), initial IOP, the extent of newly formed vessels in iris and/or angle, previous treatments, preexisting complications, treatments, final LogMAR VA, final IOP, concurrent medications, and follow-up duration. IOP was measured using a Goldmann applanation tonometer in the presence of concurrent medication.

2.5. Primary and Secondary Outcome Measurements. The primary outcome of the present study was final IOP. Secondary outcomes were LogMAR VA and number of concurrent medications. NVG cause-specific final IOP, LogMAR VA, and patient backgrounds were evaluated in the present study. IOP and the number of concurrent medications at 4 months after each treatment, including stand-alone antiVEGF, additional PRP, PPV, and LEC, were also analyzed. Further, long-term IOP prognosis associated with anti-VEGF 
TABLE 1: Cause-specific NVG patient backgrounds. NVG patients with proliferative diabetic retinopathy (PDR) were younger and had a higher pre-treatment PRP ratio. NVG patients with retinal vein occlusion (RVO) had a higher incidence of angle closure glaucoma and higher IOP than other groups. Hyphema occurred more frequently in NVG patients with ocular ischemic syndrome (OIS).

\begin{tabular}{|c|c|c|c|}
\hline Causative ocular ischemic disease & PDR & RVO & OIS \\
\hline Number of eyes & 134 & 29 & 18 \\
\hline Age (years) & $60.1 \pm 11.4$ & $72.2 \pm 15.2^{*}$ & $71.8 \pm 11.3^{*}$ \\
\hline Both eyes affected $(n)$ & $38(39.6 \%)$ & $0(0 \%)$ & $1(5.9 \%)$ \\
\hline Initial LogMAR VA & $1.34 \pm 1.06$ & $2.43 \pm 1.02$ & $1.98 \pm 1.40$ \\
\hline Initial IOP $(\mathrm{mmHg})$ & $36.4 \pm 13.8$ & $42.4 \pm 13.8^{*}$ & $35.0 \pm 11.9$ \\
\hline \multicolumn{4}{|l|}{ Criteria } \\
\hline Rubeosis group & $17(12.7 \%)$ & $0(0 \%)$ & $3(16.7 \%)$ \\
\hline Open-angle NVG group & $72(53.7 \%)$ & $12(41.4 \%)$ & $8(44.4 \%)$ \\
\hline Angle-closure NVG group & $45(33.6 \%)$ & $17(58.6 \%)^{*}$ & $7(38.9 \%)$ \\
\hline \multicolumn{4}{|l|}{ Previous treatment } \\
\hline PRP & $81(60.4 \%)$ & $8(27.6 \%)^{*}$ & $5(27.8 \%)^{*}$ \\
\hline PPV & $27(20.1 \%)$ & $2(6.9 \%)$ & $21(72.4 \%)$ \\
\hline \multicolumn{4}{|l|}{ Preexisting complication } \\
\hline Hyphema & $7(5.2 \%)^{\dagger}$ & $2(6.9 \%)^{\dagger}$ & $6(33.3 \%)$ \\
\hline $\mathrm{VH}$ & $39(29.1 \%)$ & $5(17.2 \%)$ & $4(22.2 \%)$ \\
\hline Follow-up (months) & $\begin{array}{c}26.2 \pm 22.1 \\
4.0-81.1\end{array}$ & $\begin{array}{c}17.6 \pm 18.8 \\
4.0-70.1\end{array}$ & $\begin{array}{c}16.5 \pm 13.0 \\
4.0-40.0\end{array}$ \\
\hline
\end{tabular}

PDR, proliferative diabetic retinopathy; RVO, retinal vein occlusion; OIS, ocular ischemic syndrome; VA, visual acuity; NVG, neovascular glaucoma; PRP, panretinal laser photocoagulation; $\mathrm{PPV}$, pars plana vitrectomy, $\mathrm{VH}$, vitreous hemorrhage; $\mathrm{CF}=\log 0.004 ; \mathrm{HM}=\log 0.002 ; \mathrm{SL}=\log 0.001$.

Mean \pm SD, Steel-Dwass test; ${ }^{*} P<0.05$ for PDR, ${ }^{\dagger} P<0.05$ for OIS.

and intravitreal injection combination therapy within 2 weeks of other treatments was also evaluated. Finally, we conducted multivariate statistical analyses to identify IOP prognostic factors of NVG.

2.6. Statistical Analyses. Comparisons between causespecific NVG patient groups and treatment groups were assessed by one-way repeated measures analysis of variance (ANOVA) and the Steel-Dwass test. IOP after each treatment was analyzed using the Kaplan-Meier test (end point; IOP > $21 \mathrm{mmHg}$ ). After-treatment IOP with or without anti-VEGF combination therapy was analyzed using the paired- $t$ test for pre-treatment IOP. Long-term prognosis was compared between the presence and absence of anti-VEGF combination therapy using the Mann-Whitney $U$ test and Kaplan-Meier methods (end point; IOP $>21 \mathrm{mmHg}$ ). Log-rank test and Cox proportional-hazards models were created to identify prognostic factors of NVG using final IOP $>21 \mathrm{mmHg}$ as the study end point. Causative disease (PDR, RVO, and OIS), angle closure, previous treatments (PRP, PPV), preexisting complications (hyphema, vitreous hemorrhage), and treatments (additional PRP, PPV, LEC, and anti-VEGF agents) were included as covariates. IOP, age, LogMAR VA, and follow-up durations were presented as means \pm SD. $P<0.05$ was considered statistically significant. All statistical analyses were performed using SPSS Statistics 23 (IBM, New York) and JMP11 (SAS Institute Inc., Cary, NC).

\section{Results}

3.1. Patients. We enrolled 142 patients (181 eyes) with NVG due to ocular ischemic disease. Underlying retinal diseases included PDR in 134 eyes, RVO in 29, and OIS in 18. The mean follow-up duration was $23.8 \pm 18.8$ months (range, 4.0 81.1 months). All patients at initial visits had nonperfusion areas (NPA) on FA. At the final follow-up visit, all patients were confirmed to have no evidence of NPA on FA following treatment, with IOP $\leq 21 \mathrm{mmHg}$ observed in 125 (72.3\%) eyes. No serious adverse events were observed with any treatments in the present study.

3.2. Causes and Prognosis. The mean follow-up duration was $26.2 \pm 22.1$ months (range, 4.0-81.1 months) in PDR patients, $17.6 \pm 18.8$ months (range, 4.0-70.1 months) in RVO patients, and $16.5 \pm 13.0$ months (range, 4.0-40.0 months) in OIS patients. NVG patients with PDR were younger and had a higher PRP ratio $(81 / 134,60.4 \%)$ than the RVO $(8 / 29,27.6 \%)$ and OIS groups (5/18, 27.8\%). NVG patients with RVO had a greater closed-angle ratio (angle closure NVG group, 17/29, $58.6 \%)$ and higher IOP $(42.4 \pm 13.8 \mathrm{mmHg})$ than the PDR $(45 / 134,33.6 \%, 36.4 \pm 13.8 \mathrm{mmHg})$ and OIS groups $(7 / 18$, $38.9 \%$, $35.0 \pm 11.9 \mathrm{mmHg}$ ). Patients in the NVG with OIS group had a higher incidence of hyphema $(6 / 18,33.3 \%)$ than other groups (Table 1). In the analysis of NVG cause-specific final IOP, NVG patients with PDR had lower IOP (20.7 \pm $14.2 \mathrm{mmHg})$ than the NVG with RVO $(27.3 \pm 14.2 \mathrm{mmHg})$ and OIS (26.0 $\pm 15.3 \mathrm{mmHg}$ ) groups (Figure 2 ). The majority of NVG patients had substantially lower final LogMAR VA values (1.71 \pm 1.55$)$; however, the NVG with PDR group had better LogMAR VA values $(1.39 \pm 1.45)$ compared with the those of the RVO $(2.69 \pm 1.43)$ and OIS $(2.39 \pm 1.68)$ groups (Figure 3). All vitreous hemorrhages were surgically removed and did not lead to vision loss. Severe vision loss cases (final 
TABLE 2: NVG patient backgrounds according to treatment received. The majority of patients in the LEC group had stage 3 NVG and had previously received other treatments, such as adequate PRP, stand-alone anti-VEGF therapy, and PPV. Approximately half (41.2\%) of patients that received frequent stand-alone anti-VEGF treatment had previously received repeated anti-VEGF injections.

\begin{tabular}{|c|c|c|c|c|}
\hline Treatments & $\begin{array}{c}\text { Anti-VEGF stand-alone } \\
\text { therapy }\end{array}$ & Additional PRP & PPV & LEC \\
\hline Number of treatments & 17 & 89 & 28 & 32 \\
\hline Anti-VEGF combination therapy $(n)$ & - & 49 & 15 & 3 \\
\hline Pre-treatment IOP $(\mathrm{mmHg})$ & $36.1 \pm 12.5$ & $36.1 \pm 13.5$ & $33.7 \pm 13.9$ & $35.0 \pm 8.1$ \\
\hline \multicolumn{5}{|l|}{ Criteria } \\
\hline Rubeosis group & $1(5.9 \%)$ & $9(10.1 \%)$ & $4(14.3 \%)$ & $1(3.1 \%)$ \\
\hline Open-angle NVG group & $10(58.8 \%)$ & $51(57.3 \%)$ & $12(42.9 \%)$ & $8(25.0 \%)$ \\
\hline Angle-closure NVG group & $6(35.3 \%)$ & $12(32.6 \%)$ & $12(42.9 \%)$ & $23(71.9 \%)^{*}$ \\
\hline \multicolumn{5}{|l|}{ Previous treatment } \\
\hline Anti-VEGF stand-alone therapy & $7(41.2 \%)^{*}$ & $1(1.1 \%)$ & $3(10.7 \%)^{\dagger}$ & $23(71.9 \%)^{*}$ \\
\hline PRP & $17(100.0 \%)^{*}$ & $(44.9 \%)$ & $(85.7 \%)$ & $32(100.0 \%)^{*}$ \\
\hline PPV & $4(23.5 \%)$ & $0(0 \%)$ & $0(0 \%)$ & $7(21.9 \%)$ \\
\hline LEC & $1(5.9 \%)$ & $0(0 \%)$ & $0(0 \%)$ & $1(3.1 \%)$ \\
\hline $\begin{array}{l}\text { Follow-up } \\
\text { (pre-treatment, months) }\end{array}$ & $\begin{array}{l}2.5 \pm 2.8^{*} \\
0.0 \text { to } 8.3\end{array}$ & $\begin{array}{c}0.8 \pm 3.0 \\
0.0 \text { to } 22.3\end{array}$ & $\begin{array}{l}3.2 \pm 7.9^{*} \\
1.0 \text { to } 33.3\end{array}$ & $\begin{array}{l}7.2 \pm 11.5^{*} \\
0.0 \text { to } 48.8\end{array}$ \\
\hline $\begin{array}{l}\text { Follow-up } \\
\text { (post-treatment, months) }\end{array}$ & $\begin{array}{c}21.0 \pm 19.7 \\
4.1-53.2\end{array}$ & $\begin{array}{c}25.4 \pm 21.6 \\
4.0-81.8\end{array}$ & $\begin{array}{c}25.0 \pm 14.3 \\
5.1-56.0\end{array}$ & $\begin{array}{c}24.5 \pm 22.6 \\
4.3-60.7\end{array}$ \\
\hline
\end{tabular}

PRP, panretinal laser photocoagulation; PPV, pars plana vitrectomy, LEC, trabeculectomy with mitomycin C.

Mean \pm SD, Steel-Dwass test ${ }^{*} P<0.05$ for PDR, ${ }^{\dagger} P<0.05$ for OIS.

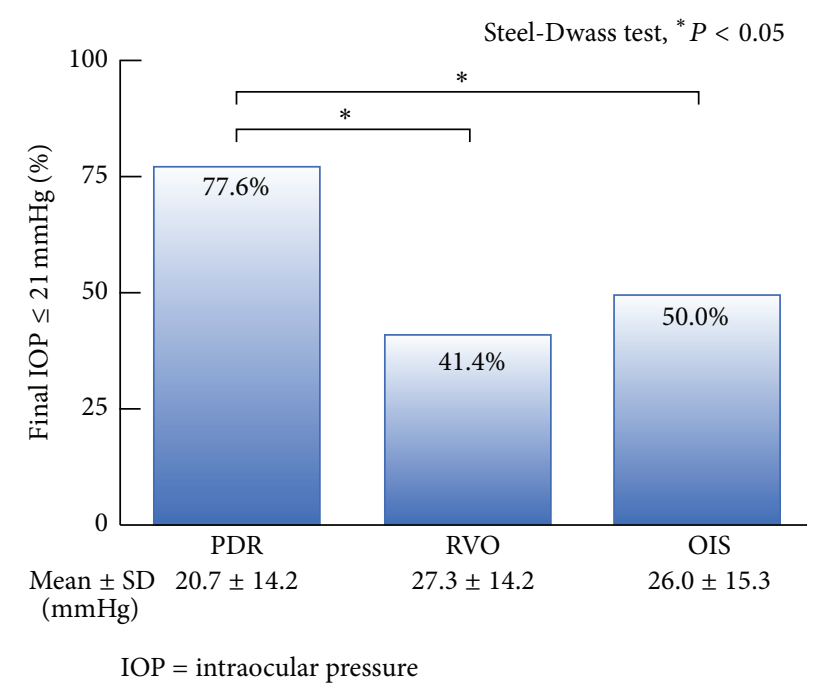

FIGURE 2: Final IOP values according to NVG causation. NVG patients with PDR had better IOP values than others.

LogMAR VA, 1.0) were 49.2\% (66/134) in PDR, 86.2\% (25/29) in RVO, and $66.7 \%(12 / 18)$ in OIS; all of them had optic atrophy. The causes for the modest vision loss (final LogMAR VA, 0.3 to 1.0 ) were macular edema or corneal edema.

3.3. Treatments and Prognosis. The mean follow-up durations pre- and post-treatment are shown in Table 2. PRP was administered to all patients who received stand-alone antiVEGF therapy. Approximately half $(7 / 17,41.2 \%)$ of these patients had previously received anti-VEGF injections (mean

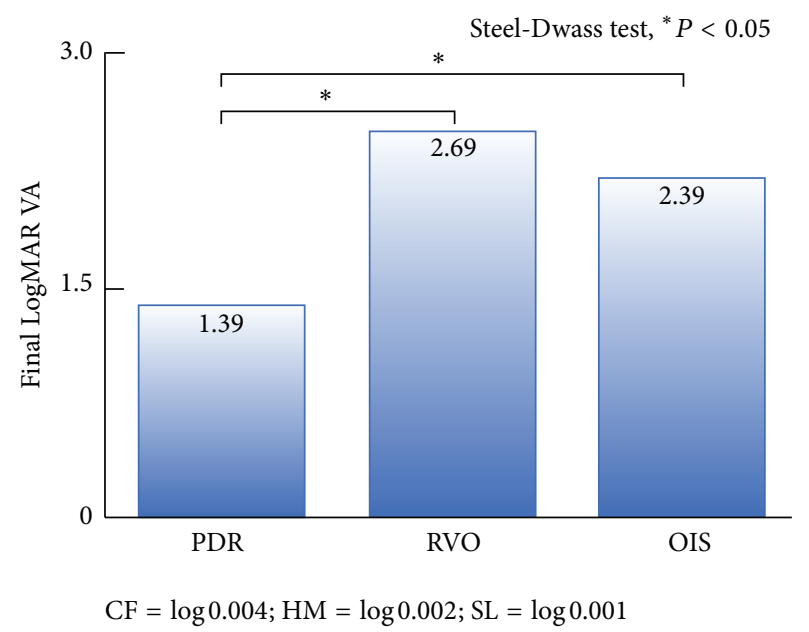

FIGURE 3: Final LogMAR VA values according to NVG causation. The majority of NVG patients had substantially lower final LogMAR VA; however, NVG patients with PDR had comparatively better final LogMAR VA than others.

number of injections, $11.1 \pm 10.4$, range, 3-31; mean duration, $2.0 \pm 1.1$ months, range 0.7-3.9 months). Forty-nine patients (55.1\%) in the additional PRP group, 15 (53.6\%) in the PPV group, and 3 (9.4\%) in the LEC group received anti-VEGF combination therapy. We performed LEC at a median time of $7.2 \pm 11.5$ months after initial visits. Twenty-three patients $(71.9 \%)$ in the LEC group had angle closure glaucoma. Patients in the LEC group had previously received anti-VEGF therapy $(23 / 32,71.9 \%)$, PPV $(7 / 32,21.9 \%)$, and adequate 


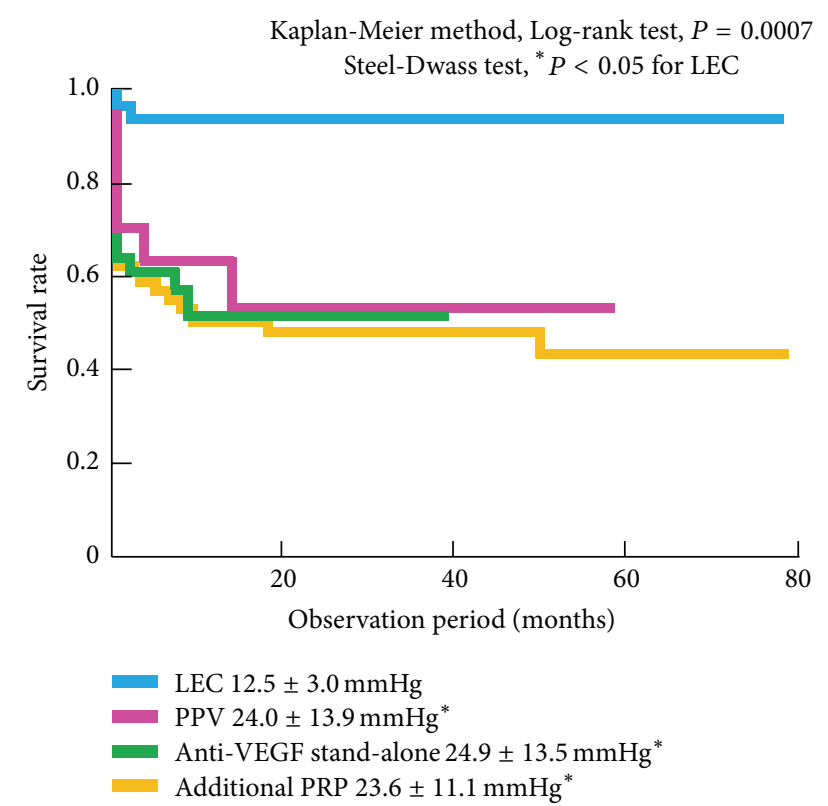

FIGURE 4: IOP progression according to by NVG treatment. LEC had the strongest continuous hypotensive effect, resulting in persistent declines in IOP $(\leq 21 \mathrm{mmHg})$ in more than $90 \%$ of patients. IOP often increased several months after treatment, except following LEC.

additional PRP (32/32, 100.0\%). Only 1 patient (3.1\%) in the LEC group underwent repeat surgery (Table 2). We compared IOP and the number of concurrent medications at 4 months after each treatment. All treatments had a significant effect on IOP. LEC had the strongest hypotensive effect among all the treatments, resulting in persistent declines in IOP in $93.8 \%(30 / 32)$ of patients (mean $24.5 \pm 22.6$ months; range, 4.3-60.7 months) (Figure 4). IOP progression and bleb survival rate after LEC had comparable Kaplan-Meier curves (data not shown). Stand-alone anti-VEGF therapy, additional PRP, and PPV also resulted in decreased IOP; however, IOP often increased several months after these treatments (Figure 4). LEC was associated with the lowest use of concurrent medications (Figure 5).

3.4. Anti-VEGF Combination Therapy and Prognosis. All treatments with or without anti-VEGF combination therapy had a significant effect on IOP. No significant differences in the post-treatment IOP were observed between patients treated with or without anti-VEGF combination therapy (Figure 6). When we examined long-term IOP prognosis after additional PRP and LEC using univariate analysis, no differences were observed between patients treated with or without anti-VEGF combination therapy (Figure 7).

3.5. Multivariate Statistics and Prognosis. Finally, we evaluated factors influencing final IOP using multivariate statistics. The results are shown in Table 3. Angle closure was found to have the greatest effect on final IOP (hazard ratio 3.059; 95\% confidence interval 1.898-4.916), followed by PDR (0.759; 0.391-0.930).

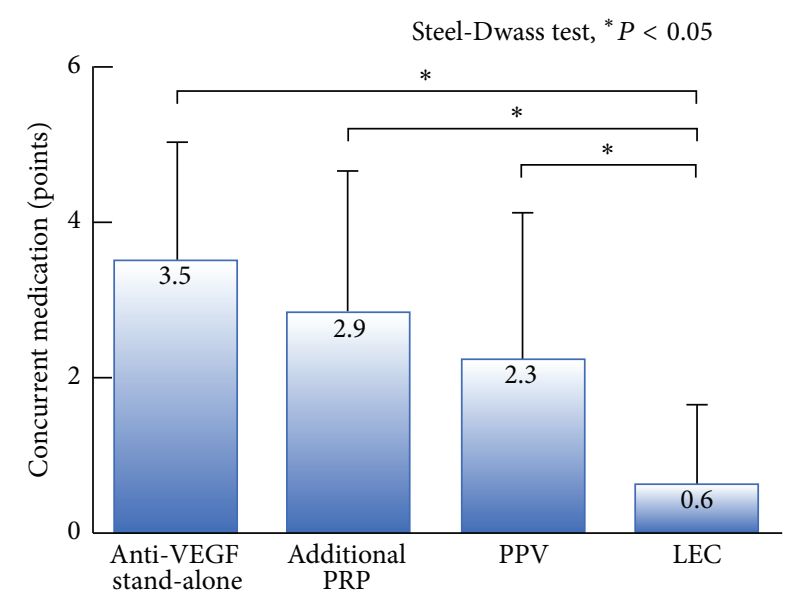

FIGURE 5: Number of concurrent medications according to NVG treatment received. LEC was associated with the lowest number of concurrent medications compared with other treatments. Concurrent medications were weighted as follows: systemic acetazolamide, 2 points; eye drops, 1 point; and mixed eye drops, 2 points.

\section{Discussion}

In this study, major cause of irreversible severe visual loss was optic atrophy. NVG with PDR had a better prognosis in terms of IOP and LogMAR VA at the final visit. Many PDR patients could escape optic atrophy because of higher PRP ratio and comparatively restricted ischemic retinal areas at the initial visit than others. Patients with NVG as a result of RVO with broad ischemia had a greater angle closure ratio, higher IOP, and worse VA prognosis.

Regarding treatment-specific prognosis, LEC had the strongest hypotensive effect compared to other treatments, with long-term decreases in IOP maintained in $93.8 \%$ of patients. During this study duration, we were unable to evaluate superior anti-VEGF compounds that have since become available, such as aflibercept, and devices, such as the tube surgical treatment option. Our superior LEC-IOP outcomes compared with those of previous studies [20] may be due to the high bleb survival rate as a result of lack of active neovascularization despite angle closure. Adequate PRP resulted in the resolution of NPA on fluorescein angiography in those patients. Full PRP followed by LEC is known to have efficacy in reducing elevated IOP associated with NVG [21]. In addition, LEC contributes to the quality of life of NVG patients by requiring the lowest combination eye drops compared to other treatments. Although other treatments may decrease IOP in the short-term, IOP often increases several months after treatment. Previous studies have reported that NVG often recurs within 1 year of treatment [22]. While additional PRP with anti-VEGF therapy and additional PRP combined with surgery are accepted as important treatments, angle closure NVG group is thought to require LEC. Approximately half of patients in the standalone anti-VEGF therapy group required repeated injections in the present study. Anti-VEGF intravitreal injections are reported to have efficacy in inducing the regression of new vessels, although this effect appears to be temporary [15, 22]. 


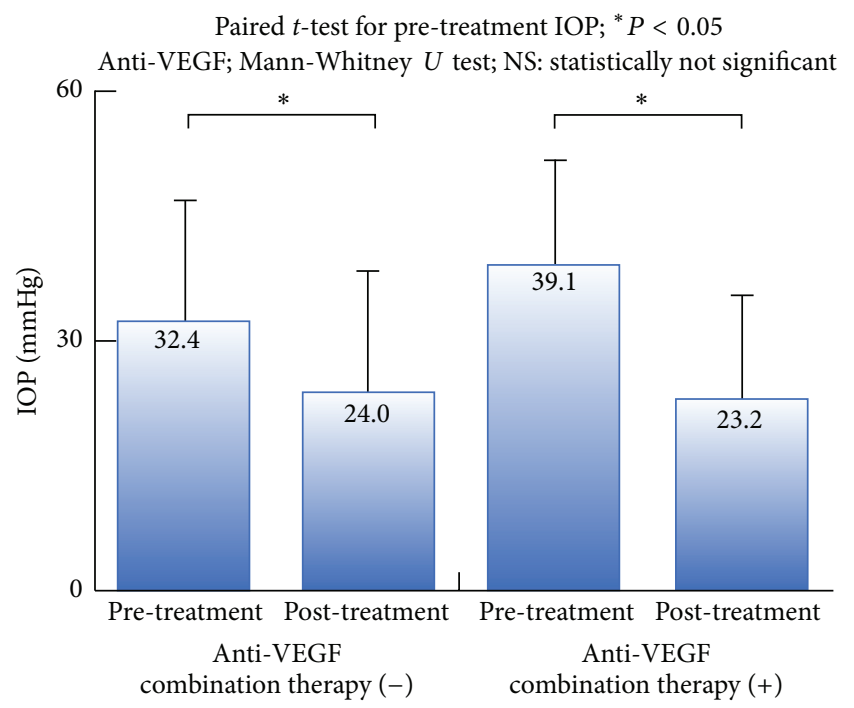

(a) Additional PRP

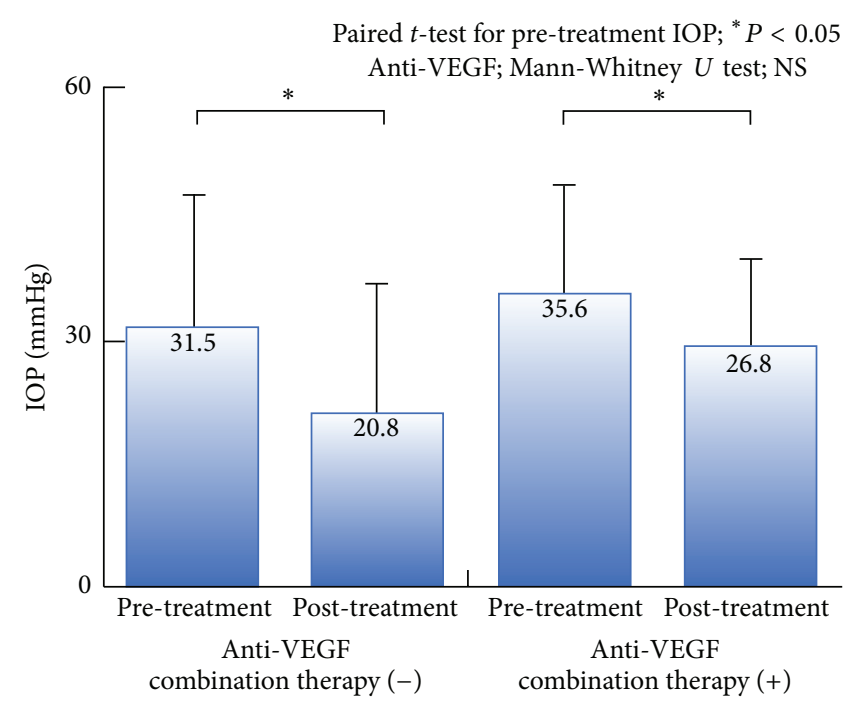

(b) PPV

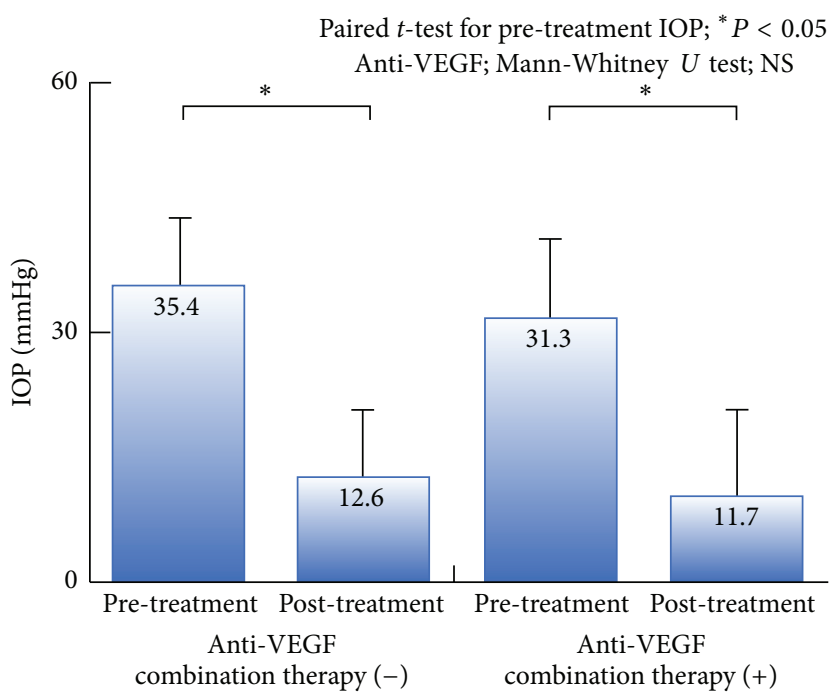

(c) LEC

FIgURE 6: Post-treatment IOP with or without anti-VEGF combination therapy. All treatments with or without anti-VEGF combination therapy had a significant effect on IOP. No significant differences in post-treatment IOP were observed between patients treated with or without anti-VEGF combination therapy.

Intravitreal injection of anti-VEGF agents in patients with NVG reportedly causes reduced vascular permeability, decreased inflammatory reaction, loss of vascular function, and endothelial cell degeneration $[14,16]$. On iris-angle angiography, dye leakage on fluorescein angiography is decreased after intravitreal injection of anti-VEGF agents. Vascular structures in the iris and angle can be observed with indocyanine angiography; however, intravitreal injection of anti-VEGF agents has no effect on these structures despite reports indicating the disappearance of newly formed vessels examined using a slit lamp [17]. Histopathological changes in the trabecular meshwork in NVG following intravitreal injection of anti-VEGF agents revealed that vascular endothelial cells were still present in the trabecular meshwork and fenestrations disappeared [14]. Therefore, repeated stand-alone
anti-VEGF injections are necessary to control IOP in NVG eyes with residual retinal ischemia. In the present study, the repeated stand-alone anti-VEGF group consisted of a small number of who refused adequate PRP and LEC due to poor general condition or for psychological reasons.

Anti-VEGF combination therapy was found to have no effect on the prognosis of NVG prognosis in terms of IOP control in the present study. The IOP prognosis of PPV with anti-VEGF combination therapy was worse than that without anti-VEGF combination therapy according to the results of univariate analysis. This finding may be attributable to the use of anti-VEGF combination therapy in severe cases of retinal neovascularization in the present study. A previous study reported IVB increased surgical success rates by decreasing risk of perioperative bleeding [23]; however, other studies 


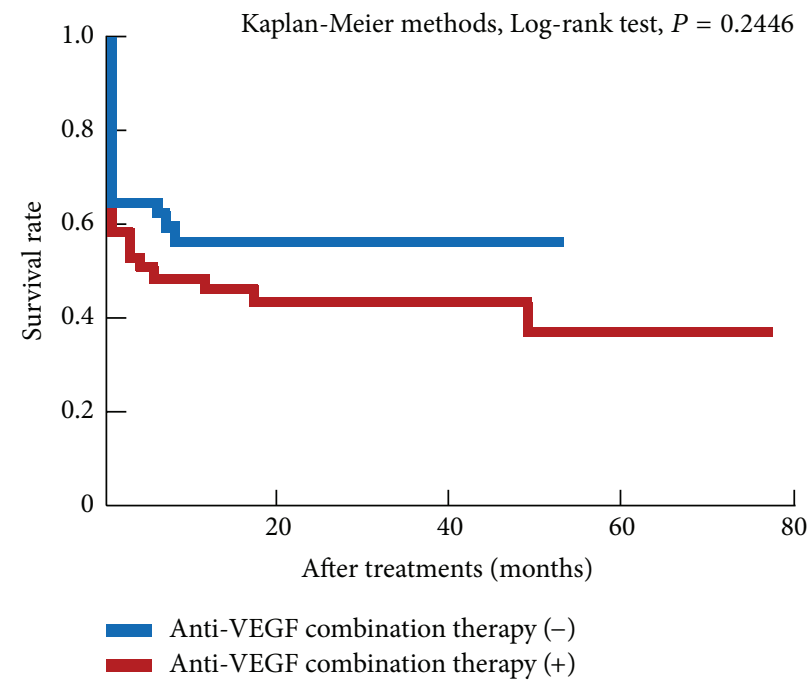

(a) Additional PRP

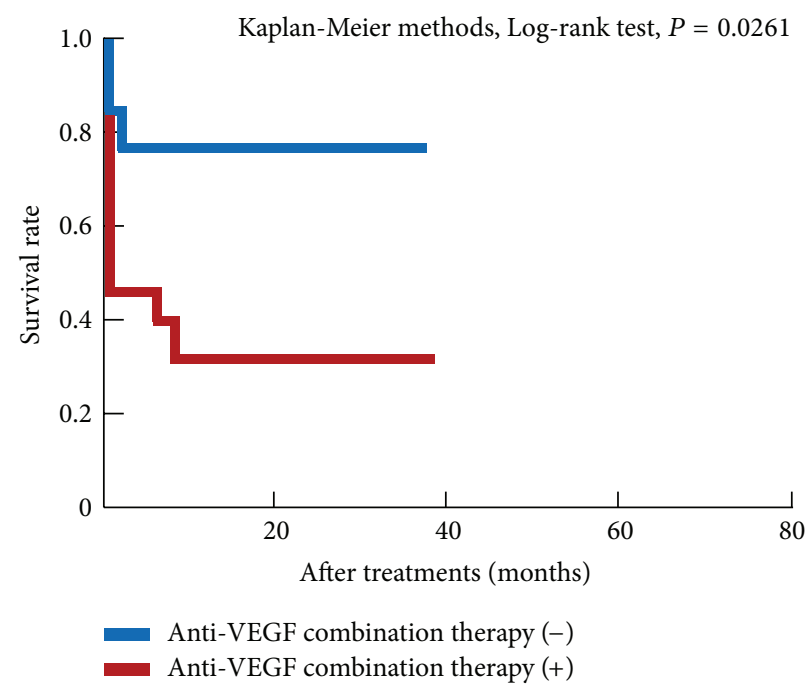

(b) PPV

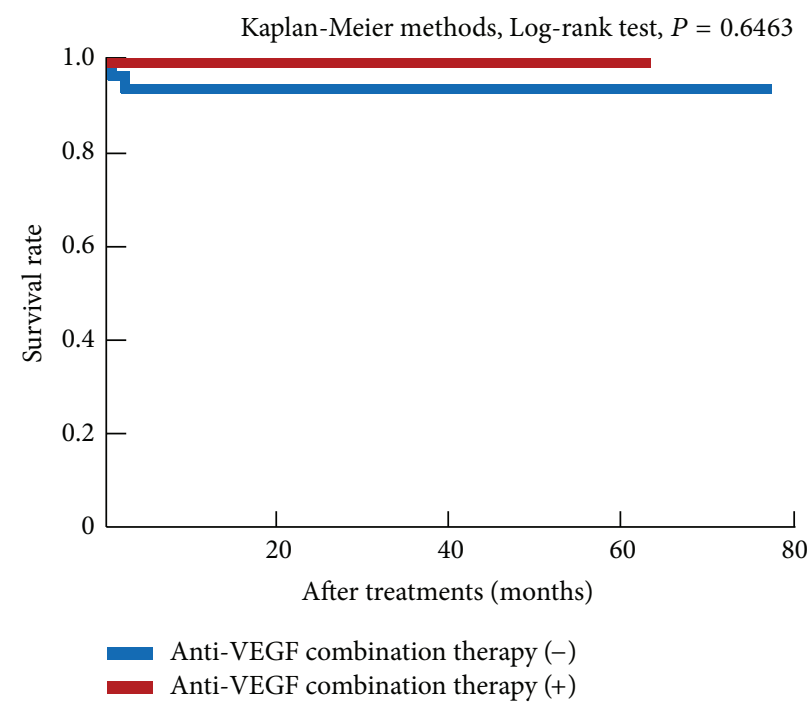

(c) LEC

FIGURE 7: Long-term prognosis of each treatment with or without anti-VEGF combination therapy (univariate analysis). Anti-VEGF combination therapy had no positive impact on long-term prognosis.

have that IVB does not improve long-term prognosis $[15,18]$. The efficacy of anti-VEGF combination therapy in improving surgical IOP outcomes remains controversial; however, it remains an accepted therapy for reducing perioperative surgical complications [18].

The present study has certain limitations. We were unable to perform a randomized study due to the retrospective design. We are planning a prospective investigator initiated trial in the future. Furthermore, we cannot try other now available superior anti-VEGF compounds [8] such as aflibercept [19] and devises like the tube surgical option of treatment [24-26] due to disapproval at that time. In addition, as LEC was performed after various treatments, including adequate PRP (100\%), anti-VEGF stand-alone therapy (71.9\%), and PPV (21.9\%), it should be noted that extensive preparation is necessary for the success of LEC. Our follow-up duration after the last LEC treatment (mean $24.5 \pm 22.6$ months) may have been inadequate for accurate assessment of long-term prognosis.

\section{Conclusions}

We summarize our present results from the retrospective study involving $142 \mathrm{NVG}$ patients. Angle closure was found to have the greatest effect on NVG-IOP prognosis. Therefore, LEC with survival blebs after other adequate treatments, including anti-VEGF treatments, appears to be the effective treatment for NVG. Anti-VEGF combination therapy had no effect on long-term NVG-IOP prognosis but is recommended prior to angle closure. In patients where the target IOP is not obtained following adequate PRP with/without the use of anti-VEGF agents, early LEC may improve the prognosis of NVG-IOP this time, and a high index of suspicion based on patient history and early recognition of high risk eyes are 
TABLE 3: Prognostic factors influencing final IOP in patients with NVG (multivariate statistics). Log-rank test and Cox proportional-hazards models were created to identify prognostic factors of NVG using final IOP $>21 \mathrm{mmHg}$ as the study end-point. Angle-closure was associated with a 3-fold worsening in NVG-IOP prognosis. Patients with NVG with PDR had relatively better prognosis than those with NVG induced by other causes.

\begin{tabular}{|c|c|c|c|}
\hline Covariates & Hazard ratio & 95\% confidence interval & $\begin{array}{c}\text { Log-rank test } \\
\left({ }^{*} P<0.05\right)\end{array}$ \\
\hline Angle-closure NVG group & 3.059 & $1.898-4.916$ & $0.0002^{*}$ \\
\hline Causative disease, PDR & 0.759 & $0.391-0.930$ & $0.0002^{*}$ \\
\hline Treatments, LEC & 0.412 & $0.251-0.667$ & 0.0809 \\
\hline Causative disease, RVO & - & - & $0.0123^{*}$ \\
\hline Causative disease, OIS & - & - & $0.0384^{*}$ \\
\hline Previous treatments, PRP & - & - & 0.1667 \\
\hline Previous treatments, PPV & - & - & 0.2717 \\
\hline Pre-existing complications, hyphema & - & - & 0.3930 \\
\hline Pre-existing complications, VH & - & - & 0.8108 \\
\hline Treatments, anti-VEGF therapy & - & - & 0.3128 \\
\hline Treatments, additional PRP & - & - & 0.3642 \\
\hline Treatments, PPV & - & - & 0.9287 \\
\hline
\end{tabular}

NVG, neovascular glaucoma; PDR, proliferative diabetic retinopathy; LEC, trabeculectomy with mitomycin C; RVO, retinal vein occlusion; OIS, ocular ischemic syndrome; PRP, panretinal laser photocoagulation; PPV, pars plana vitrectomy; VH, vitreous hemorrhage.

crucial for favorable long-term outcomes. Moreover, NVG treatments are rapidly evolving with time. The randomized prospective study including newest IOP-lowering devises and drugs may be necessary for next prospective study in the future.

\section{Competing Interests}

The authors declare that they have no competing interests.

\section{References}

[1] S. S. Hayreh, "Neovascular glaucoma," Progress in Retinal and Eye Research, vol. 26, no. 5, pp. 470-485, 2007.

[2] J. A. Sivak-Callcott, D. M. O’Day, J. D. M. Gass, and J. C. Tsai, "Evidence-based recommendations for the diagnosis and treatment of neovascular glaucoma," Ophthalmology, vol. 108, no. 10, pp. 1767-1800, 2001.

[3] S. Gartner and P. Henkind, "Neovascularization of the iris (rubeosis iridis)," Survey of Ophthalmology, vol. 22, no. 5, pp. 291-312, 1978.

[4] M. Kim, C. Lee, R. Payne, B. Y. J. T. Yue, J.-H. Chang, and H. Ying, "Angiogenesis in glaucoma filtration surgery and neovascular glaucoma: a review," Survey of Ophthalmology, vol. 60, no. 6, pp. 524-535, 2015.

[5] T. Kubota, A. Tawara, Y. Hata, A. Khalil, and H. Inomata, "Neovascular tissue in the intertrabecular spaces in eyes with neovascular glaucoma," British Journal of Ophthalmology, vol. 80, no. 8, pp. 750-754, 1996.

[6] K. Kovacs, K. V. Marra, G. Yu et al., "Angiogenic and inflammatory vitreous biomarkers associated with increasing levels of retinal ischemia," Investigative Ophthalmology \& Visual Science, vol. 56, no. 11, pp. 6523-6530, 2015.

[7] K. V. Chalam, V. S. Brar, and R. K. Murthy, "Human ciliary epithelium as a source of synthesis and secretion of vascular endothelial growth factor in neovascular glaucoma," JAMA Ophthalmology, vol. 132, no. 11, pp. 1350-1354, 2014.

[8] N. Kitnarong, C. Sriyakul, and S. Chinwattanakul, "A prospective study to evaluate intravitreous ranibizumab as adjunctive treatment for trabeculectomy in neovascular glaucoma," Ophthalmology and Therapy, vol. 4, no. 1, pp. 33-41, 2015.

[9] J. Lüke, K. Nassar, M. Lüke, and S. Grisanti, "Ranibizumab as adjuvant in the treatment of rubeosis iridis and neovascular glaucoma-results from a prospective interventional case series," Graefe's Archive for Clinical and Experimental Ophthalmology, vol. 251, no. 10, pp. 2403-2413, 2013.

[10] S. Ishibashi and H. Kondo, "Effect of intravitreal bevacizumab injection on iris and iridocorneal angle neovascularization in neovascular glaucoma," Journal of UOEH, vol. 37, no. 4, pp. 299304, 2015.

[11] Y. Saito, T. Higashide, H. Takeda, S. Ohkubo, and K. Sugiyama, "Beneficial effects of preoperative intravitreal bevacizumab on trabeculectomy outcomes in neovascular glaucoma," Acta Ophthalmologica, vol. 88, no. 1, pp. 96-102, 2010.

[12] T. Wakabayashi, Y. Oshima, H. Sakaguchi et al., "Intravitreal bevacizumab to treat iris neovascularization and neovascular glaucoma secondary to ischemic retinal diseases in 41 consecutive cases," Ophthalmology, vol. 115, no. 9, pp. 1571-1580.e3, 2008.

[13] J. R. SooHoo, L. K. Seibold, and M. Y. Kahook, "Recent advances in the management of neovascular glaucoma," Seminars in Ophthalmology, vol. 28, no. 3, pp. 165-172, 2013.

[14] T. Kubota, R. Aoki, Y. Harada et al., "Trabecular meshwork in neovascular glaucoma eyes after the intravitreal injection of bevacizumab," British Journal of Ophthalmology, vol. 93, no. 4, pp. 557-558, 2009.

[15] Y. Sugimoto, H. Mochizuki, H. Okumichi et al., "Effect of intravitreal bevacizumab on iris vessels in neovascular glaucoma patients," Graefe's Archive for Clinical and Experimental Ophthalmology, vol. 248, no. 11, pp. 1601-1609, 2010.

[16] N. Yoshida, T. Hisatomi, Y. Ikeda et al., "Intravitreal bevacizumab treatment for neovascular glaucoma: histopathological 
analysis of trabeculectomy specimens," Graefe's Archive for Clinical and Experimental Ophthalmology, vol. 249, no. 10, pp. 1547-1552, 2011.

[17] S. Ishibashi, A. Tawara, R. Sohma, T. Kubota, and N. Toh, "Angiographic changes in iris and iridocorneal angle neovascularization after intravitreal bevacizumab injection," Archives of Ophthalmology, vol. 128, no. 12, pp. 1539-1545, 2010.

[18] Y. Takihara, M. Inatani, T. Kawaji et al., "Combined intravitreal bevacizumab and trabeculectomy with mitomycin $\mathrm{C}$ versus trabeculectomy with mitomycin C alone for neovascular glaucoma," Journal of Glaucoma, vol. 20, no. 3, pp. 196-201, 2011.

[19] M. Tang, Y. Fu, Y. Wang et al., "Efficacy of intravitreal ranibizumab combined with Ahmed glaucoma valve implantation for the treatment of neovascular glaucoma Glaucoma," BMC Ophthalmology, vol. 16, no. 1, article 7, 2016.

[20] Y. Takihara, M. Inatani, M. Fukushima, K. Iwao, M. Iwao, and H. Tanihara, "Trabeculectomy with mitomycin C for neovascular glaucoma: prognostic factors for surgical failure," American Journal of Ophthalmology, vol. 147, no. 5, pp. 912-918.el, 2009.

[21] S. A. Al Obeidan, E. A. Osman, S. A. Al-Amro, D. Kangave, and A. M. Abu El-Asrar, "Full preoperative panretinal photocoagulation improves the outcome of trabeculectomy with mitomycin C for neovascular glaucoma," European Journal of Ophthalmology, vol. 18, no. 5, pp. 758-764, 2008.

[22] A. Goto, M. Inatani, T. Inoue et al., "Frequency and risk factors for neovascular glaucoma after vitrectomy in eyes with proliferative diabetic retinopathy," Journal of Glaucoma, vol. 22, no. 7, pp. 572-576, 2013.

[23] S. Kojima, M. Inatani, K. Shobayashi, A. Haga, T. Inoue, and H. Tanihara, "Risk factors for hyphema after trabeculectomy with mitomycin C," Journal of Glaucoma, vol. 23, no. 5, pp. 307-311, 2014.

[24] H. B. Hwang, J. W. Han, H. B. Yim, and N. Y. Lee, "Beneficial effects of adjuvant intravitreal bevacizumab injection on outcomes of Ahmed glaucoma valve implantation in patients with neovascular glaucoma: systematic literature review," Journal of Ocular Pharmacology and Therapeutics, vol. 31, no. 4, pp. 198203, 2015.

[25] S. J. Gedde, J. C. Schiffman, W. J. Feuer, L. W. Herndon, J. D. Brandt, and D. L. Budenz, "Treatment outcomes in the tube versus trabeculectomy (TVT) study after five years of followup," American Journal of Ophthalmology, vol. 153, no. 5, pp. 789e2, 2012.

[26] Z. Li, M. W. Zhou, W. Wang et al., "A prospective comparative study on neovascular glaucoma and non-neovascular refractory glaucoma following Ahmed glaucoma valve implantation," Chinese Medical Journal, vol. 127, no. 8, pp. 1417-1422, 2014. 


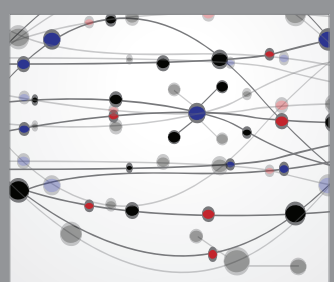

The Scientific World Journal
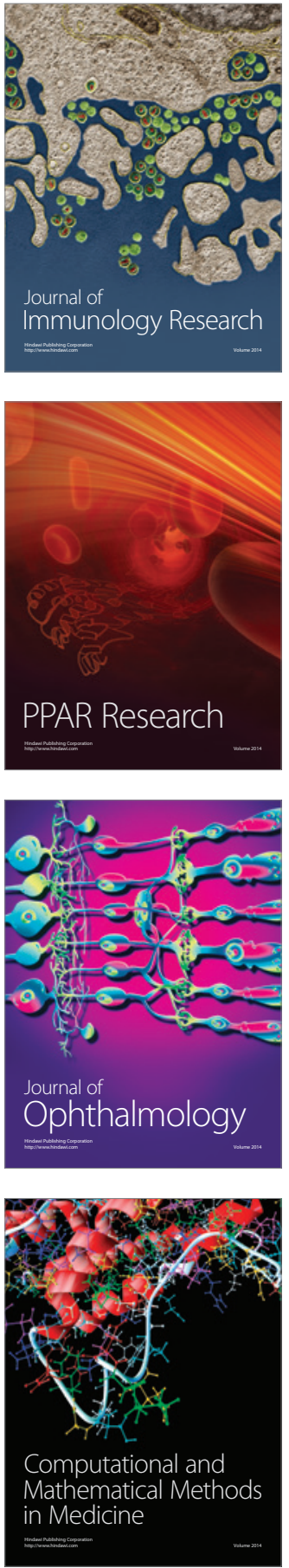

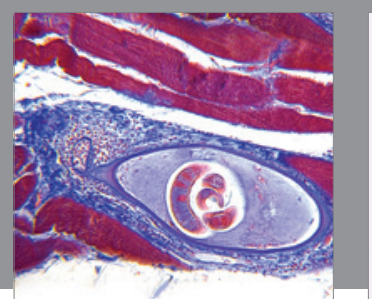

Gastroenterology Research and Practice

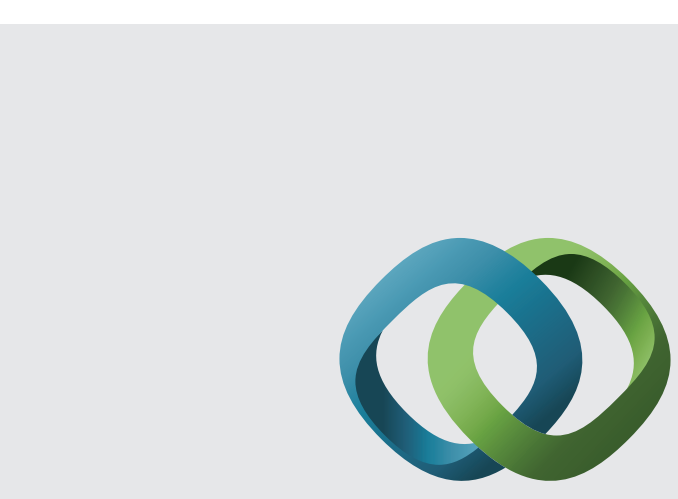

\section{Hindawi}

Submit your manuscripts at

http://www.hindawi.com
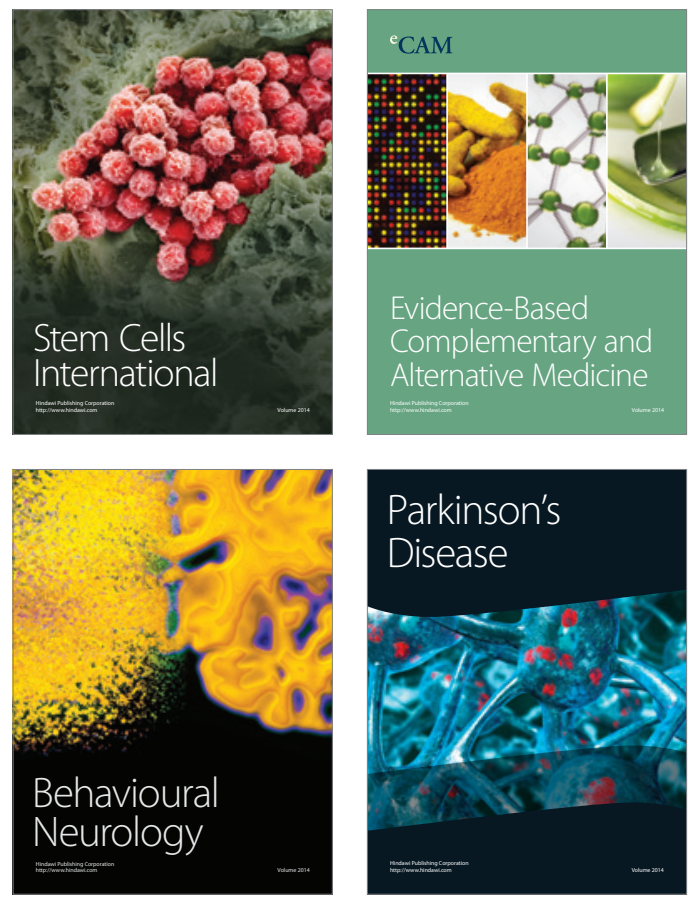
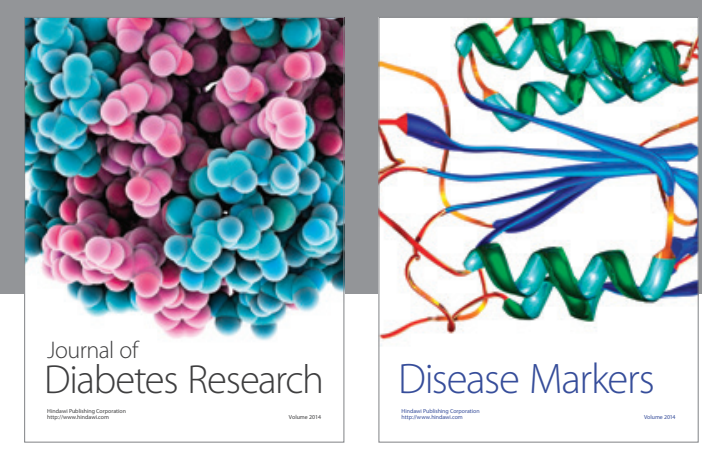

Disease Markers
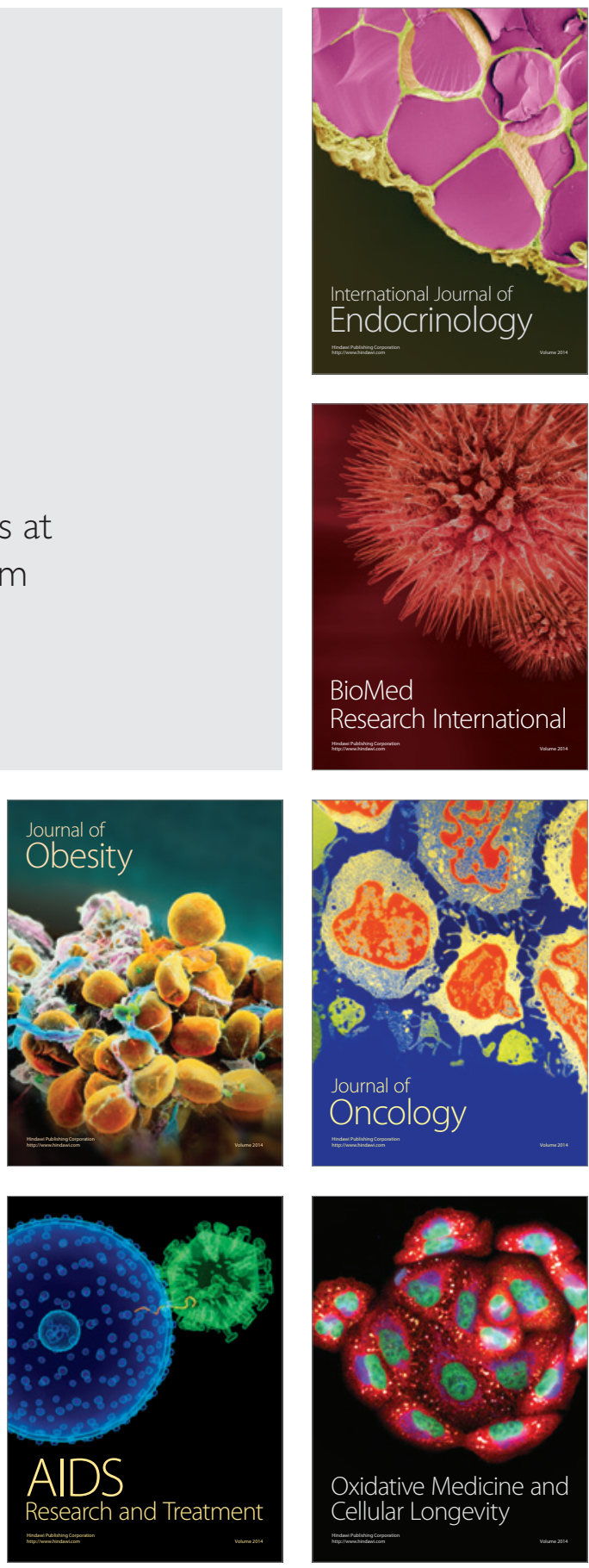